19 Revue d'histoire du XIXe siècle

Société d'histoire de la révolution de 1848 et des

révolutions du XIXe siècle

$62 \mid 2021$

Ancêtres

\title{
Erwan POINTEAU-LAGADEC, Le Club des hachichins. Du mythe à la réalité
}

Jean-Jacques Yvorel

\section{OpenEdition}

\section{Journals}

Édition électronique

URL : https://journals.openedition.org/rh19/7708

DOI : $10.4000 /$ rh 19.7708

ISSN : $1777-5329$

Éditeur

La Société de 1848

\section{Édition imprimée}

Date de publication : 20 juin 2021

Pagination : $278-280$

ISSN : 1265-1354

Référence électronique

Jean-Jacques Yvorel, "Erwan POINTEAU-LAGADEC, Le Club des hachichins. Du mythe à la réalité », Revue d'histoire du XIXe siècle [En ligne], 62 | 2021, mis en ligne le 17 août 2021, consulté le 26 août 2021. URL : http://journals.openedition.org/rh19/7708 ; DOI : https://doi.org/10.4000/rh19.7708

Ce document a été généré automatiquement le 26 août 2021.

Tous droits réservés 


\title{
Erwan POINTEAU-LAGADEC, Le Club des hachichins. Du mythe à la réalité
}

\author{
Jean-Jacques Yvorel
}

\section{RÉFÉRENCE}

Erwan POINTEAU-LAGADEC, Le Club des hachichins. Du mythe à la réalité, Paris, Le Manuscrit, 2020, 109 p., 13,90€.

Dans la petite collection dirigée par Myriam Tsikounas consacrée aux addictions, Erwan Pointeau-Lagadec a entrepris de revisiter « un pilier » de l'histoire des drogues : Le Club des hachichins de Théophile Gautier. Ce texte, publié le $1^{\mathrm{er}}$ février 1846 dans la très respectable Revue des Deux Mondes, rend compte des expériences de consommation de cannabis de l'auteur du Roman de la momie. Ces fantasias, comme il les nomme, se déroulent dans les appartements du peintre Boissard à l'hôtel Pimodan sur l'île SaintLouis. Doit-il être lu comme un témoignage sur le fonctionnement d'une société secrète d'amateurs de cannabis ou comme une pure invention littéraire ? Telle est la question de départ de cette courte enquête.

Dans un premier chapitre l'auteur situe le contexte dans lequel les soirées de l'hôtel Pimodan ont vu le jour. Après avoir souligné la place de la campagne d'Égypte dans la circulation du produit, il relève l'intérêt croissant d'une poignée de médecins et d'artistes pour le haschisch. Du côté des médecins il cite Louis Rémy Aubert-Roche, Alexandre Brierre de Boismont et bien sûr Jacques-Joseph Moreau dit Moreau de Tour, auteur $\mathrm{Du}$ hachisch et de l'aliénation mentale. Du côté des littérateurs, outre Théophile Gautier, il évoque Alphonse Karr. Il s'agit bien "d'une poignée » d'intéressés et nullement d'une consommation de masse, même si la littérature médicale nous offre quelques autres exemples d'expérimentation ${ }^{1}$. Il aurait peut-être fallu ajouter que cette curiosité ne concernait pas que le haschisch, mais toutes les substances psychoactives. De plus, ces expérimentations, qu'elles soient médicales ou "artistiques " ne sont l'objet d'aucun opprobre. Seul l'excès est dénoncé. Le très austère Brierre de Boismont, 
par exemple, qualifie l'utilisation de certains stimulants « lorsqu'ils ne dépassent pas la mesure » de " conditions des ferments nécessaires aux créations de l'esprit ${ }^{2}$ ». Ce cadre répond, semble-t-il, pour partie à la question. Nul besoin de constituer une société secrète pour se livrer à une pratique qu'aucune loi ne réprime et que la morale ne condamne pas encore.

Dans un second chapitre, Erwan Pointeau-Lagadec, soutient l'idée que le Club des hachichins désigne moins un groupe de personnes ou une institution qu'un espacetemps : l'hôtel Pimodan de 1845 à 1849. Le tout-Paris Bohème et romantique fréquente régulièrement cet hôtel du 17 quai d'Anjou où résident Boissard et Baudelaire. Le peintre y organise fréquemment des dîners ou des concerts et quelques fois des "dégustations" de haschisch qui constituent la matière du texte de Gautier. "Le départ du peintre [...] mettra un terme à cette période de réjouissances, laissant aux habitués des lieux et aux étrangers de passage d'agréables souvenirs et une nostalgie certaine. » (p. 44). Surtout il met fin « à une dynamique d'appropriation du haschisch et de ses propriétés par un groupe donné d'individus» (p.54) et signe donc la « dissolution » du Club des hachichins.

Dans le troisième chapitre, Erwan Pointeau-Lagadec, se penche sur la composition de ce groupe. Au terme d'une analyse de l'ensemble des témoignages disponibles, il révèle le fonctionnement $\mathrm{du}$ «club». Nous trouvons d'abord un noyau dur composé de Théophile Gautier, Fernand Boissard de Boisdenier et du docteur Jacques-Joseph Moreau dit Moreau de Tour. Viennent ensuite trois habitués, les écrivains Alphonse Karr et Charles Baudelaire ainsi que le peintre Ernest Meissonier. Enfin une dizaine de visiteurs plus occasionnels parmi lesquels les docteurs Louis Aubert-Roche et Edouard Talien de Cabarrus, Honoré de Balzac et, sans certitude, Eugène Delacroix, forment l'ultime cercle. Aucune femme au sein du noyau dur ou dans le premier cercle, une présence très incertaine parmi les «occasionnels", le Club des hachichins est indiscutablement une organisation virile.

Nous avons avec le Club des hachichins une structure de sociabilité des plus classiques ${ }^{3}$ que l'auteur va interroger dans un quatrième chapitre. Il montre l'écart entre les réunions des protagonistes de l'hôtel Pimodan et le fonctionnement d'un cercle au $\mathrm{XIX}^{\mathrm{e}}$ siècle. Il élimine toute référence à un club républicain et souligne le refus de tout engagement politique de Gautier, tenant de l'art pour l'art ${ }^{4}$. La référence à un club plus ou moins secret et mystérieux n'est qu'un procédé littéraire, " une variation ludique sur le thème de la conspiration » (p. 81).

Dans un ultime chapitre, Erwan Pointeau-Lagadec cherche à expliquer «la genèse du mythe ». Comment ces quelques soirées d'expérimentation du haschisch qui ne concernaient au mieux qu'une grosse dizaine de personnes, ont pu être considérées comme le point de départ d'une intoxication de masse et comme l'origine d'un problème social majeur. La première raison qui explique l'importance prise par un texte qui aurait pu, comme bien des articles de la Revue des deux mondes, tomber dans l'oubli, c'est la nostalgie des participants aux soirées organisées par Boissard. Ils ne manquent pas dans leurs écrits ultérieurs d'évoquer les fantasias et contribuent à une véritable patrimonialisation du texte de Gautier. La seconde raison, l'auteur la voit dans l'écho des Paradis artificiels de Baudelaire vus, à tort, comme une incitation à la consommation de drogue. Enfin, il incrimine la recherche historique française qui aurait laissé s'impatroniser un "double statut imaginaire de membre d'une société secrète et de précurseur de la toxicomanie moderne» (p.101). Cette dernière 
explication ne nous a pas convaincus. D'abord parce que les textes auxquels l'auteur renvoie ne sont pas des textes relevant méthodologiquement du travail historique. Ensuite parce qu'il nous semble que les travaux qui sont du ressort de l'histoire ont bien montré que le " problème des drogues » n'est pas né, en France, avec le Club des Hachichins mais avec le développement de la morphinomanie, intoxication essentiellement iatrogène et bien plus tardive, puis avec la fumerie d'opium liée à la colonisation de l'Indochine ${ }^{5}$. Le Club des hachichins appartient au "moment cognitif » des drogues, moment important certes, mais qui ne saurait être vu comme fondateur de la «toxicomanie moderne». Quant à l'historiographie française de l'usage des stupéfiants, elle n'est pas aussi indigente que semble le dire l'auteur. Certes, il reste bien des recherches à mener, mais trois thèses d'histoire, qui couvrent toute la période contemporaine $^{6}$, ont bien déblayé le terrain.

7 Dans une conclusion qui ouvre une réflexion sur les temps présents, l'auteur souligne, après bien d'autres chercheurs, qu'ils soient historiens, juristes, sociologues ou addictologues, la nécessité d'une évolution législative.

\section{NOTES}

1. Dans sa thèse de 1839 , le docteur F. Lallemand reconnait consommer du haschisch pour son plaisir.

2. Alexandre Brierre de Boismont, Des hallucinations, ou Histoire raisonnée des apparitions, des visions, des songes, de l'extase, du magnétisme et du somnambulisme, Paris, G. Baillière, 1862 [1 $1^{\text {re }}$ éd. 1845], p. 205-206. Comme stimulants, il cite le protoxyde d'azote, l'opium et le haschisch. Par ailleurs, il écrit dans le numéro de janvier 1852 des Annales médico-psychologiques un article dénonçant les dangers de l'abus de haschisch.

3. Noyau dur, premier cercle, participants occasionnels, cette trilogie se retrouve dans la plupart des associations qu'elles soient de loisir ou humanitaire, dans les «bandes de jeunes » et plus largement dans les groupes amicaux.

4. Dans son introduction à ses poèmes Émaux et camés (publiés en 1852), faisant allusion aux événements révolutionnaires, Théophile Gautier écrit: "Sans prendre garde à l'ouragan / Qui fouettait mes vitres fermées, / Moi, j'ai fait Émaux et Camées. »

5. Jean-Jacques Yvorel, Les Poisons de l'esprit. Drogues et drogués en France au XIXe siècle, Paris, Quai Voltaire, 1992.

6. Ibid.; Emmanuelle Retaillaud-Bajac, Les Paradis perdus. Drogues et usagers de drogues dans la France de l'entre-deux-guerres, Rennes, Presses universitaires de Rennes, 2009; Alexandre Marchant, L'Impossible prohibition. Drogues et toxicomanie en France 1945-2017, Paris, Perrin, 2018. 